\title{
Growth of High Quality Mg-Doped GaAs by Molecular Beam Epitaxy and Its Properties
}

\author{
Jong Su KIM, D. Y. LEE and I. H. BAE \\ Department of Physics, Yeungnam University, Kyongsan 712-749

\begin{abstract}
J. I. Lee, S. K. Noh, Jin Soo Kim, Gu Hyun Kim, Seungil Ban, Se-Kyung Kang and S. M. Kim
Materials Evaluation Center, Korea Research Institute of Standards and Science, Taejon 305-340
\end{abstract} \\ J. Y. LEEM* and Minhyon JEON \\ Department of Optical Engineering, Inje University, Kimhae 621-749 \\ J. S. SON \\ School of Architecture, Environment and Life Science, Kyungwoon University, Kumi 730-850
}

(Received 1 November 2000, in final form 21 July 2001)

\begin{abstract}
The high quality GaAs epitaxial layers with various $\mathrm{Mg}$ doping levels were grown by molecular beam epitaxy (MBE) by changing substrate temperature and III/V beam equivalent pressure (BEP) ratio. The carrier concentration and mobility obtained from room temperature Hall measurement are of $2 \times 10^{15} \sim 1.2 \times 10^{20} \mathrm{~cm}^{-3}$ and $239 \sim 11 \mathrm{~cm}^{2} / \mathrm{V} \cdot \mathrm{sec}$, respectively. During growth of GaAs epitaxial layer, the reflection high-energy electron diffraction (RHEED) pattern showed streaky line regardless of doping level. From the photoluminescence (PL) spectra, band to acceptor transitions $(e-A)$ are dominant at $10 \mathrm{~K}$ and its ionization energy is $27.6 \mathrm{meV}$. The high-resolution x-ray diffraction measurements (DCXRD) with different carrier concentration showed high structural quality. This is the first observation for high quality GaAs epitaxial layer grown by MBE even at high Mg doping level.
\end{abstract}

\section{INTRODUCTION}

Magnesium ( $\mathrm{Mg}$ ) has been studied as a shallow acceptor in III-V semiconductors since Cho et al. first reported that it could be used as a p-type dopant for GaAs grown by molecular beam epitaxy (MBE) $[1,2]$. In 1982, Wood et al. studied that the dependence of $\mathrm{Mg}$ incorporation into GaAs as a function of the substrate temperature (Ts) and concluded that $\mathrm{Mg}$ incorporation coefficient ( $K i$ ) approached to unity below $T s$ of $500{ }^{\circ} \mathrm{C}$, and got reduced at high $T s$ of $600{ }^{\circ} \mathrm{C}$ by three orders of magnitude to $0.3 \times 10^{-3}[3]$. From their results, the free hole concentration reduced from $\sim 10^{18}$ to $\sim 10^{16} \mathrm{~cm}^{-3}$ range. These substrate temperature dependent characteristics restrict the use of Mg. However, currently, GaAs molecular beam epitaxy (MBE) layers grown at low temperatures $(\mathrm{LT})\left(200 \sim 250{ }^{\circ} \mathrm{C}\right)$ are attracting much interest due to the minimizing of several common device problems such as backgating and sidegating to realize high-

${ }^{*}$ E-mail : jyleem@ijnc.inje.ac.kr speed device applications. The low-dimensional system such as quantum dots (QDs) grown at temperature below $500{ }^{\circ} \mathrm{C}$ has also been widely studied [4]. $\mathrm{Mg}$ is also commonly used as a p-type dopant for GaN $[5,6]$ and related material, such as InGaN [7], AlGaN [8] even at high growth temperatures. There are also well-known advantages of $\mathrm{Mg}$ doping such as satisfactory nontoxic, and non-carcinogenic alternative to Beryllium (Be). From these reasons, $\mathrm{Mg}$ could be suggested as very acceptable p-type dopant for III-V compound semiconductor.

To the best of our knowledge, no experimental result for high carrier concentration has been presented so far for $\mathrm{Mg}$ doped GaAs grown by MBE. Two different techniques such as growth temperature and Arsenic (As) beam equivalent pressure (BEP) at same Mg cell temperature is utilized in order to obtain high-quality $\mathrm{Mg}$ doped GaAs epilayer. The crystal qualities and properties were investigated by reflection high-energy electron diffraction (RHEED), Hall measurement, photoluminescence $(\mathrm{PL})$, and double crystal x-ray diffraction (DCXRD). From the experimental results, we obtained 


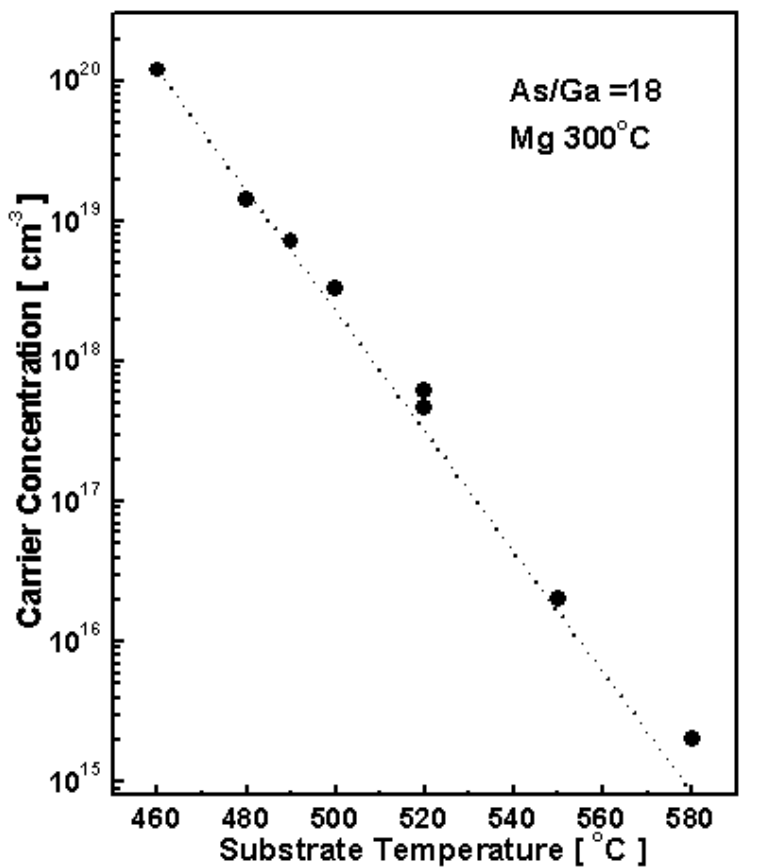

Fig. 1. Carrier concentration of Mg-doped GaAs layer grown at different substrate temperatures, reflecting an decreasing $\mathrm{Mg}$ incorporation coefficient as Ts increases.

high quality $\mathrm{Mg}$ doped GaAs with a higher hole concentration and mobility rather than those reported before and have believed that these techniques are one of the promising way for the growth of high quality Mg doped GaAs epitaxial layer.

\section{EXPRIMENT}

The samples studied in this work were grown on GaAs(100) substrates by RIBER 32P molecular beam epitaxy (MBE), which is equipped with a $12 \mathrm{keV}$ RHEED system. After the surface oxide layer is removed by in situ annealing under As flux at a substrate temperature of $540{ }^{\circ} \mathrm{C}$, a $300 \mathrm{~nm}$ undoped GaAs buffer layer is deposited at $580{ }^{\circ} \mathrm{C}$, and the Mg-doped $500 \mathrm{~nm}$ GaAs layer is grown with different growth temperature ranging from 460 to $580^{\circ} \mathrm{C}$ and different $\operatorname{As} \operatorname{BEP}\left(7.4 \times 10^{-6}\right.$ $\sim 1.6 \times 10^{-5}$ Torr). At the end of the $\mathrm{Mg}$ doped layer growth, RHEED of all samples clearly showed a streaky $(2 \times 4)$ pattern. Hall measurements were carried out on the Mg doped GaAs samples using Van der Pauw method at room temperature and determined a hole concentration and mobility. In order to realize the ohmic contact for Hall measurement, indium (In) dots with a diameter of $1 \mathrm{~mm}$ was fabricated using a thermal evaporator. After the thermal evaporations, the samples were annealed for $60 \mathrm{~s}$ at $450{ }^{\circ} \mathrm{C}$ in a $\mathrm{N}_{2}$ atmosphere by the proximity capping method. The photoluminescence (PL) measurement was carried out in a closed cycle He cryostat with

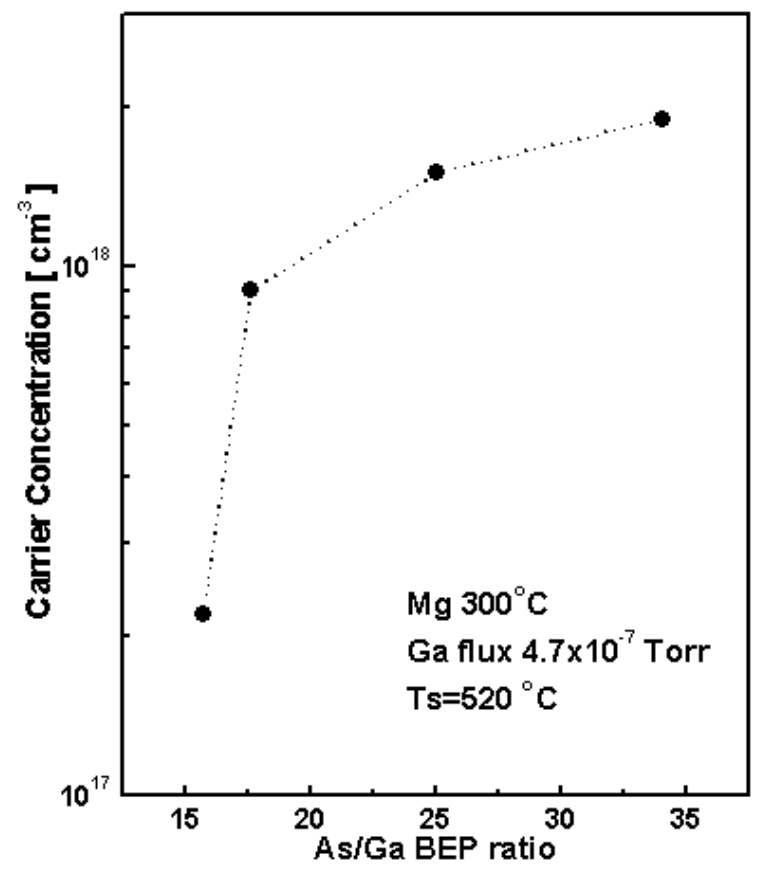

Fig. 2. Carrier concentrations of $\mathrm{Mg}$-doped GaAs layer grown at different III/V beam equivalent pressure (BEP) ratio.

a focused He-Ne gas laser beam $(\lambda=632.8 \mathrm{~nm})$ with its maximum excitation power of $30 \mathrm{~mW}$. The PL signal is dispersed by a $0.75 \mathrm{~m}$ monochromator and detected with a water-cooled photomultiplier tube using lock-in techniques. x-ray diffraction (DCXRD) using high-resolution triple axis diffraction method was performed to evaluate crystal qualities of Mg doped GaAs.

\section{RESULTS AND DISCUSSION}

During the growth of Mg-doped GaAs epilayers, the RHEED lines for all samples showed a streaky pattern regardless of doping levels.

Figure 1 shows free hole concentration of Mg-doped GaAs layers grown at different substrate temperatures $(T s)$. We obtained various hole concentration with 2 $\times 10^{15} \sim 1.2 \times 10^{20} \mathrm{~cm}^{-3}$ and in particular, the carrier concentration for GaAs epilayer grown at substrate temperature of $460{ }^{\circ} \mathrm{C}$ is $1.2 \times 10^{20} \mathrm{~cm}^{-3}$, which is the highest value known so far. The carrier concentration decreased as a function of exponential, as the substrate temperature is increased. This is reflecting a decreasing $\mathrm{Mg}$ incorporation coefficient with increase in $T s$. Similar results in carrier concentration was reported by Wood et al. [3].

Figure 2 shows free hole concentration of Mg-doped GaAs layer grown at different III/V BEP ratio. The carrier concentration is increased in about one order as increase of III/V BEP ratio from 15 to 35 when Ga and $\mathrm{Mg}$ fluxes to the substrate were kept constant. 


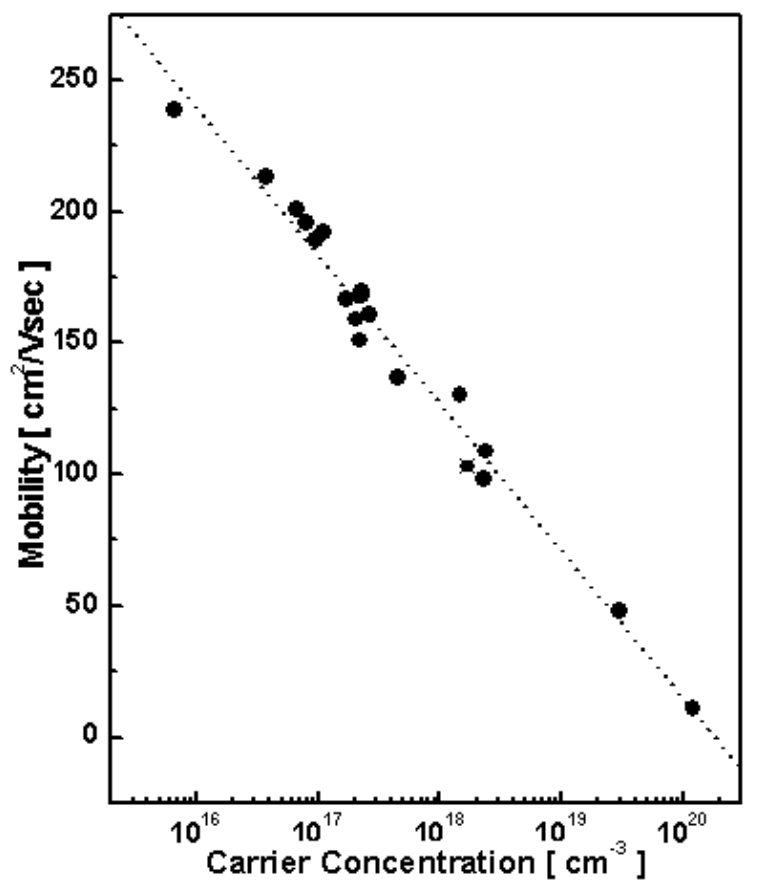

Fig. 3. Hole mobility vs. carrier concentration for $\mathrm{Mg}$ doped GaAs epilayers at room temperature.

From Fig.1 and 2, we successfully obtained various hole concentrations by changing growth parameters such as substrate temperature and III/V ratio.

Figure 3 shows hole mobility as a function of carrier concentration for $\mathrm{Mg}$ doped GaAs epitaxial layers at room temperature. The room temperature Hall mobility of these p-type epilayers varied from $239 \mathrm{~cm}^{2} / \mathrm{Vsec}$ for $\mathrm{N}_{A^{-}} \mathrm{N}_{D}=2 \times 10^{15} \mathrm{~cm}^{-3}$ to $11 \mathrm{~cm}^{2} / \mathrm{Vsec}$ for $\mathrm{N}_{A^{-}}$ $\mathrm{N}_{D}=1.2 \times 10^{20} \mathrm{~cm}^{-3}$. As the hole concentration is increased, the hole mobility is decreased due to the enhancement of ionized impurity scattering and screening effects [9].

Figure 4 shows the PL emission spectra from selected Mg-doped GaAs samples $(S 1-S 6)$ with different carrier concentration measured at $10 \mathrm{~K}$. The PL from $6.6 \times$ $10^{15}(S 1)$ and $6.6 \times 10^{16} \mathrm{~cm}^{-3}(S 2)$ GaAs samples show three emission peaks about (1) $1.4922 \mathrm{eV}$, (2) 1.5105 $\mathrm{eV}$ and (3) $1.5130 \mathrm{eV}$. These peaks were identified from temperature-dependent PL spectra (not shown here) as follows; (1) band to acceptor transitions resulting in $\mathrm{Mg}$ acceptor $(e-A),(2)$ acceptor associated emission $(g)$ and $(3)$ excitons bound to neutral acceptor $\left(A^{\circ} X\right)$ respectively [10]. The activation energy for $e-A$ is 27.6 $\mathrm{meV}$ that agrees well with the result obtained from $\mathrm{Mg}$ implanted and $\mathrm{Mg}$ doped GaAs $[2,11,12]$. The PL from the sample with carrier concentration of $2.6 \times 17^{15} \mathrm{~cm}^{-3}$ (S3) shows four emission peaks at $1.4908 \mathrm{eV}, 1.5024 \mathrm{eV}$, $1.5072 \mathrm{eV}$ and $1.5133 \mathrm{eV}$. The additional peak between $g$ and $e-A$ could be from unintentional impurities or acceptor associated emission $(g-g)$ [10]. Takeuchi et al. reported that the $g$ and $g-g$ lines are produced by ion

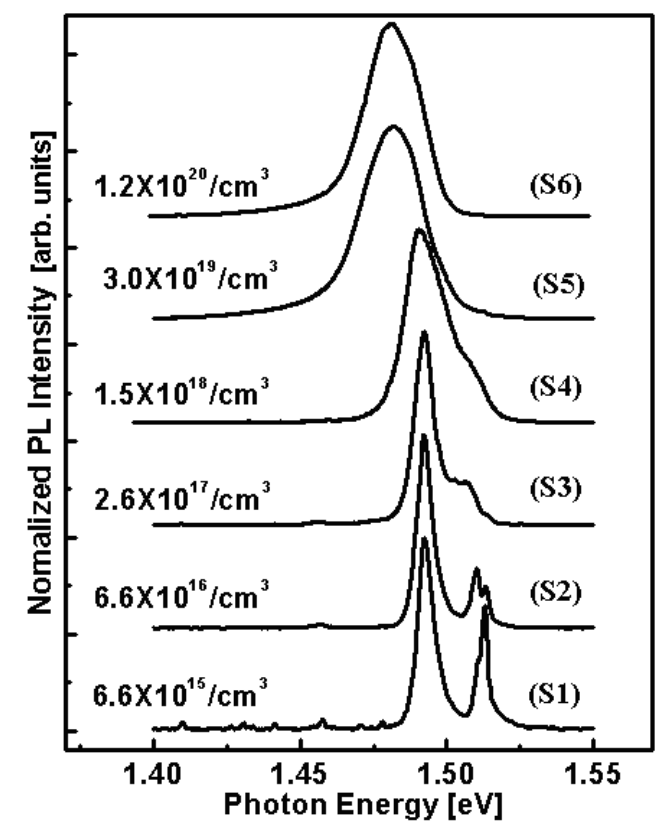

Fig. 4. Photoluminescence emission spectra obtained from Mg-doped GaAs with carrier concentration of $6.6 \times 10^{15} \sim$ $1.2 \times 10^{20} \mathrm{~cm}^{-3}$.

implantation of well-established acceptor impurities $\left(\mathrm{C}^{+}\right.$, $\mathrm{Mg}^{+}, \mathrm{Zn}^{+}$, and $\left.\mathrm{Cd}^{+}\right)$and dependent on acceptor concentration [11]. In our case, the $g$ and $g-g$ were formed in Mg-doped GaAs grown by MBE and the characteristic features are dependent on carrier concentration. Therefore, the $g$ and $g-g$ could be acceptor atoms in GaAs. The PL from the samples with high carrier concentration of $1.5 \times 10^{18}(S 4), 3.0 \times 10^{19}(S 5)$ and $1.2 \times 10^{20} \mathrm{~cm}^{-3}$ $(S 6)$ show one dominant but broad peak. The PL from $e-A$ with increase in carrier concentration up to 1.5 $\times 10^{18} \mathrm{~cm}^{-3}(S 4)$ is red-shifted leading to lowering the energy position. This is caused by a many-body effect known as bandgap renormalization, which is due to the apparent shrinkage of the bandgap induced by the heavy doping [13]. The significant point in PL spectra is rapid change in energy of $e-A$ between $S 4$ and $S 5$. With increase in acceptor concentration, the free to bound exciton first broadened because of changes in the density of states of the acceptor level density of states. As the acceptors become closer to one another, their wavefunctions begin to overlap resulting in broadening of the acceptor levels into an impurity band. When the band is so broad that it overlaps with the valence band, holes are no longer localized on the acceptors and become free carriers called Mott transition [14].

In order to confirm the crystal quality of $\mathrm{Mg}$ doped GaAs epilayers, DCXRD measurements were carried out. Figure 5 shows the x-ray rocking curves for each sample and FWHMs of DCXRD rocking curves with different carrier concentration are summarized in Fig. 6. From the DCXRD results, we did not observe significantly difference, such as peak separation from that of the sub- 


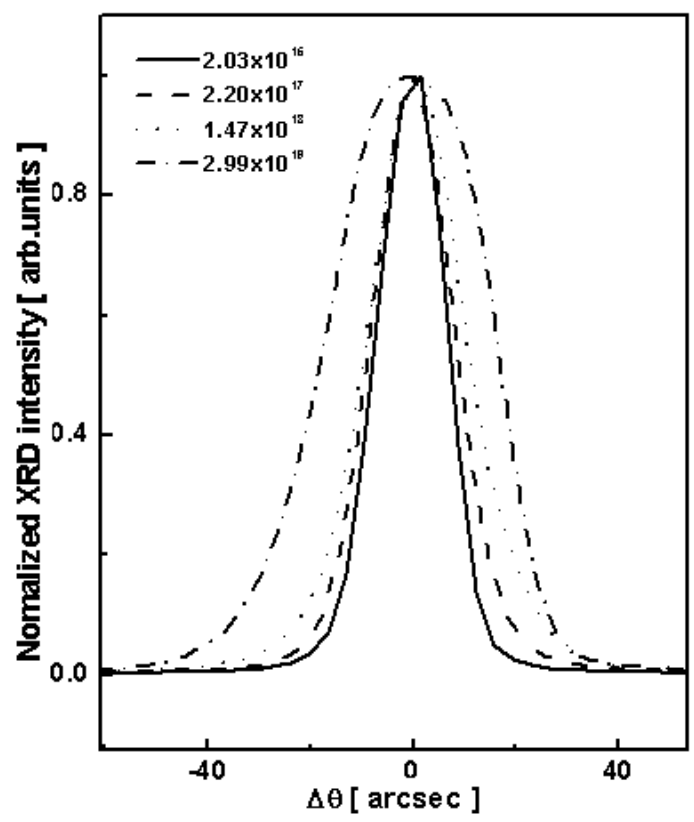

Fig. 5. X-ray rocking curves of Mg-doped (004) GaAs planes.

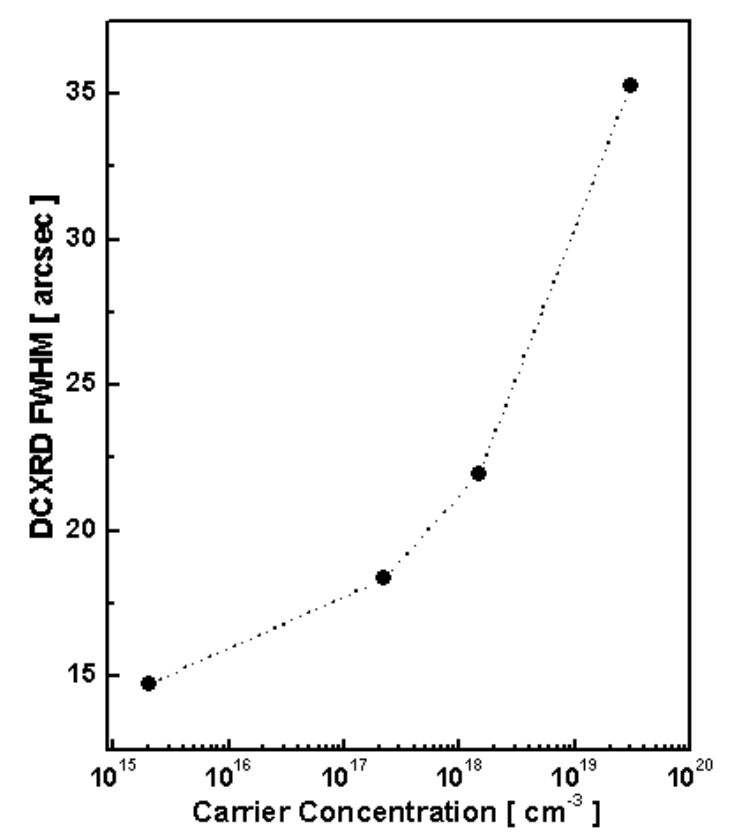

Fig. 6. FWHM of DCXRD rocking curves for Mg-doped GaAs with different carrier concentration.

strate. The FWHM of x-ray rocking curves is increased from 15 to 35 arcsec with increase in doping concentration, however, the change is very small implying that high quality GaAs epitaxial layer were grown even at high doping level by MBE. The broadening in FWHM of $\mathrm{x}$-ray rocking curves can be explained by change in lattice constant cased by the $\mathrm{Mg}$ atom, which substitutes for $\mathrm{Ga}$ atom. When the carrier concentration is increased, that is, the $\mathrm{Mg}$ incorporation in GaAs structure is enhanced, the lattice constant of host crystal is perturbed.

\section{CONCLUSIONS}

We successfully obtained high quality GaAs epitaxial layer with various hole concentrations by changing growth parameters such as substrate temperature and III/V ratio. The carrier concentration and mobility obtained from room temperature Hall measurement are of $2 \times 10^{15} \sim 1.2 \times 10^{20} \mathrm{~cm}^{-3}$ and $239 \sim 11 \mathrm{~cm}^{2} / \mathrm{Vsec}$, respectively. From the observation of RHEED pattern during growth of Mg-doped GaAs epilayers, PL and DCXRD measurement, high quality Mg doped GaAs epilayers were grown, even at very high doping concentration.

We believe these methods are one of the promising way for the deposition of high quality $\mathrm{Mg}$ doped GaAs epitaxial layer growth.

\section{REFERENCES}

[1] A. Y. Cho and M. B. Panish, J. Appl. Phys. 43, 5118 (1972).

[2] D. J. Ashen, P. J. Dean, D. T. J. Hurle, J. B. Mullin, A. M. White and P. D. Greene, J. Phys. Chem. Sol. 36, $1041(1975)$

[3] C. E. C. Wood, D. Desimone, K. Singer and G. W. Wicks, J. Appl. Phys. 53, 4230 (1982).

[4] J. S. Kim, P. W. Yu, J. Y. Leem, J. I. Lee, S. K. Noh, Jong Su Kim, S. M. Kim, J. S. Son, U. H. Lee, J. S. Yim and D. Lee, Appl. Phys. Lett. 21, 3247 (2001).

[5] J. Y. Leem, C. R. Lee, J. I. Lee, S. K. Noh, Y. S. Kwon, Y. H. Ryu and S. J. Son, J. Crystal Growth 193, 491 (1998).

[6] J. K. Kim, Y. H. Cho, J. S. Kwak, O. H. Nam. J. Lee, Y. Park, T. Kim and J. L. Lee, J. Korean Phys. Soc. 39, 23 (2001).

[7] H. Morkç, J. Korean Phys. Soc. 30, S26 (1997).

[8] M. Razeghi, P. Kung, D. Walker, E. Monroy, M. Hamilton and P. Sandvik, J. Korean Phys. Soc. 34, S234 (1999).

[9] P. Y. Yu and M. Cardona, Fundamentals of Semiconductors Physics and Materials Properties (Springer, Berlin, 1996), p. 207.

[10] Jong Su Kim, I. H. Bae, J. Y. Leem, S. K. Noh, J. I. Lee, J. S. Kim, S. M. Kim, J. S. Son and Minhyon Jeon, J. Crystal Growth 226, 52 (2001).

[11] Y. Takeuchi, Y. Makita, K. Kudo, T. Nomura, H. Tanaka, K. Irie and N. Ohnishi, Appl. Phys. Lett. 48, 59 (1986).

[12] P. W. Yu and Y. S. Park, Appl. Phys. Lett. 30, 14 (1977).

[13] G. D. Mahan, Many-Particle physics (Plenum, New York 1990).

[14] C. Kittel : Introduction to solid state phys., $6^{\text {th }}$ ed. (Wiley, New York, 1986) Chap. 10, p. 269. 